\title{
Long-Term Use of Motion-Based Video Games in Care Home Settings
}

\author{
Kathrin M. Gerling \\ University of Lincoln \\ Brayford Pool \\ Lincoln, LN6 7TS, UK \\ kgerling@lincoln.ac.uk
}

\author{
Regan L. Mandryk \\ University of Saskatchewan \\ 110 Science Place \\ Saskatoon, SK, Canada \\ regan@cs.usask.ca
}

\author{
Conor Linehan \\ University of Lincoln \\ Brayford Pool \\ Lincoln, LN6 7TS, UK \\ clinehan@lincoln.ac.uk
}

\begin{abstract}
Recent research suggests that motion-based video games have the potential to provide both mental and physical stimulation for older adults in residential care. However, little research has explored the practical challenges and opportunities that arise from integrating these games within existing schedules of activities in these contexts. In our work, we report on a qualitative enquiry that was conducted over a three month period at two long-term care facilities. Findings suggest that older adults enjoyed playing video games, and that games can be a valuable means of reintroducing challenge in late life, but that the impact of agerelated changes and impairment can influence people's ability to engage with games in a group setting. We outline core challenges in the design for care context and discuss implications of our work regarding the suitability of games as a self-directed leisure activity.
\end{abstract}

\section{INTRODUCTION}

Many older adults in Western societies depend on the care of others (e.g., family caregivers, or care facilities). Despite consistent findings that mental and physical activity are crucial for well-being [23], providing stimulating and accessible leisure activities for older adults in residential care can be difficult, due to the impact of age-related changes and impairments. Researchers have explored the potential of video games - particularly motion-based video games requiring some physical effort to play [18] - as an activity appropriate for this audience. Previous work has demonstrated positive effects of such games on the cognitive [2], physical [3] and emotional [13] well-being of older adults in long-term care. In this context, games are frequently cited as a means of fostering social interaction between older adults [26], and one that can be carried out without extensive support [20]. However, this topic has only begun to be explored [12], and the existing research is

Permission to make digital or hard copies of all or part of this work for personal or classroom use is granted without fee provided that copies are not made or distributed for profit or commercial advantage and that copies bear this notice and the full citation on the first page. Copyrights for components of this work owned by others than ACM must be honored. Abstracting with credit is permitted. To copy otherwise, or republish, to post on servers or to redistribute to lists, requires prior specific permission and/or a fee. Request permissions from Permissions@acm.org.

CHI 2015, April 18 - 23, 2015, Seoul, Republic of Korea.

Copyright 2015 ACM 978-1-4503-3145-6/15/04 ...\$15.00.

http://dx.doi.org/10.1145/2702123.2702125 relatively disjointed. For example, human-computer interaction (HCI) researchers have focused on questions of accessibility and player experience, using research methodologies derived from lab-based usability studies [10]. Researchers from other disciplines have focused primarily on health outcomes [2,3]. An aspect that has not been explored extensively is the interaction of challenges and opportunities that arise, on a practical basis, from the deployment of games in a group setting in long-term care.

In this paper, we present a qualitative enquiry into the experiences that arose from the deployment of motionbased video games at two care facilities. Weekly gaming sessions were organized for three months at two locations that differed in terms of both the level of care offered and the cognitive and physical ability of participants. Data was analyzed using Thematic Analysis [6] and revealed three main themes of interest: 1) the game experience of older adults; 2) implications of playing games in a social setting; and 3) levels of support required to engage with games. First, our findings suggest that video game playing can be a challenging and appealing activity that allows older adults to experience competence. However, the extent to which people experience the benefit of gaming is determined largely by their specific experience of age-related changes and impairments, as demonstrated by the very different experiences observed in our two study locations. Second, our results show that learning to play new games in a social (i.e., public) context may be uncomfortable for people experiencing vulnerability over their age-related changes and impairments. Third, our results show that some older adults require extensive support, both to gain access to gaming sessions and throughout play, suggesting that the value of games as self-directed activity may be limited for some groups of players.

Our work makes three contributions: (1) we provide an enquiry into the deployment of video games at two fundamentally different long-term care facilities, a senior residence that offers assisted living for older adults, and a care home that accommodates older adults with cognitive and physical impairment; (2) we discuss elements of shared video game play that may increase the vulnerability of older adults, and we address the value of shared play for community building; and (3) we identify challenges in the deployment of games in care facilities, and outline 
strategies to support designers wishing to create engaging games for older adults in long-term care.

If games are to benefit older adults in long-term care, it is crucial to examine the gaming experiences of older adults, and to develop a more detailed understanding of the potential benefits and risks of video gaming in the caregiving context to ensure that games provide positive and empowering experiences for older adults regardless of age-related changes and impairments.

\section{RELATED WORK}

In this section, we summarize existing research efforts addressing the deployment of technologies in residential care and game development for older adults.

Technology to Facilitate Caregiving and Assisted Living Technologies to facilitate caregiving and to support assisted living are an important and active area of research. Much work in the field of human-computer interaction has focused on improving the caregiving process [7], e.g., by deploying information technology to monitor older adults and thereby support caregivers [15], or the development of systems in the field of ambient-assisted living that aim to help older adults maintain independence, e.g., applications to facilitate medication routines [14].

\section{Technology to Connect and Entertain}

Other researchers have focused on the deployment of technology to improve the quality of life of older adults in residential care by fostering mental, physical and social activity. Blythe et al. [4] report on the deployment of four interactive interventions in a care home in the UK, which were designed to stimulate older adults by enhancing their awareness of their surroundings. They showed that playful applications can foster socialization between older adults and across generations by providing tickets to talk. Work by Müller et al. [19], exploring the deployment of a largescreen display in a German care home, suggests that technology can help older adults connect with each other and staff members. Finally, Lindley et al. [16] present a literature review that suggests that technology can be applied to help older adults maintain relationships with family members and friends, and outline play as one of the main areas of interest in HCI for older adults.

\section{Video Games for Older Adults}

In this context, the design of video games has been approached from two perspectives, (1) looking at how older adults interact with commercially available games, and (2) the design of video games addressing the needs of older adults from the perspective of human-computer interaction.

\section{Deploying Commercially Available Games}

Researchers have explored potential benefits of video game play on the emotional well-being of institutionalized older adults. A six-week study by Jung et al. [13] showed that video game play had a significantly higher positive impact on participants' overall well-being than traditional board games. Marston et al. [17] present a review of long-term studies that deploy commercially-available video games in residential care. Their summary shows that such games have the potential of fostering social interaction and improving the emotional well-being of older adults in residential care; however, many studies follow a quantitative approach (e.g., [13]) instead of exploring the value of games in the context of day-to-day routines of care, and do not examine the effects of play on older adults with a wide range of cognitive and physical abilities. In this context, results of an uncontrolled longitudinal quantitative study by Ulbrecht et al. [27] suggest that cognitive functioning has a profound impact on institutionalized older adults' engagement with motion-based video games. In our work, we follow up on these findings through a qualitative enquiry that investigates how the context of play and individual abilities influence older adults' engagement.

\section{Designing Games for Older Adults}

Research has addressed game design for older adults from different perspectives, exploring the value of games to entertain, to connect players, and as a tool to support therapy and rehabilitation. For example, Rice et al. [24] explore how motion-based games can foster intergenerational play between younger and older adults, showing that games have the potential to connect different generations. Uzor and Baille [28], as well as Alankus et al. [1], demonstrate that motion-based games can successfully be applied to motivate participation in physical therapy and rehabilitation in the home. Specifically investigating the use of gaming technology in group settings, Pedell et al. [21] explore the value of mobile gaming applications to connect older adults. Their results show that games may bring older adults together, but also note that "some older adults are shy in groups". Gerling et al. [10,11] investigate the design of motion-based game interfaces for older adults in residential care, focusing on the individual player experience of institutionalized older adults, as well as general considerations regarding game accessibility.

On a general level, while work on games for therapy and rehabilitation has previously addressed aspects related to the long-term engagement of older adults with games in their own home $[1,28]$ and in long-term care [27], little is known about social and contextual dynamics that develop when older adults in residential care are invited to engage with games. A first enquiry into this area is provided by Harley et al. [12] who explore how older adults in longterm care engage with Nintendo Wii Sports Bowling. In our work, we build on their findings and further explore how older adults in residential care approach games, and how individual cognitive and physical abilities as well as the social setting influence their experience.

\section{EXPLORING GAMES FOR OLDER ADULTS IN RESIDENTIAL CARE}

In our case study, we provide a qualitative enquiry into the experiences of older adults living at two long-term care facilities who were invited to engage with video games in the context of three months of weekly gaming sessions. 


\section{The Care Facilities}

We worked with two care facilities to study how different groups of older adults experience video game play; Brightwater Senior Living - referred to as 'the senior residence' - is a senior residence offering independent and assisted living apartments, and Sherbrooke Community Centre - referred to as 'the care home' - is a nursing home that provides extensive care for its residents.

Brightwater Senior Living is a newly opened, privately run senior residence in Saskatoon, SK, Canada. The residence offers independent and assisted living apartments for older adults. Most residents are independent or only experience mild age-related changes and impairments that do not have a severe impact on their ability of attending to personal needs. At the time of the study, the oldest resident was 96 years old, and the youngest resident was aged 59. The routines at the home are roughly structured by three optional meals served in a restaurant-style area (residents also have a kitchenette in their apartment), as well as morning and afternoon activities. Activities are usually facilitated by staff and include physical exercise, cultural activities (e.g., museum visits) and creative expression (e.g., writing and painting), as well as movie showings and excursions to local leisure facilities (e.g., bowling). Staff is available throughout the day to support residents based on individual needs. While most residents require light assistance (e.g., cleaning services), others experience higher levels of age-related changes that result in a need for support in personal areas (e.g., running errands).

Sherbrooke Community Centre is a government-funded care facility in Saskatoon, SK, Canada, that specializes in providing care for individuals with special needs, including older adults who experience high levels of age-related impairments, e.g., persons with dementia or complex mobility disabilities. The facility follows the Eden Alternative $^{1}$ approach to care, which emphasizes the importance of meaningful activities and community living in caregiving for older adults. It also offers palliative care and a day program. At the time of the study, the oldest resident was more than 100 years old. The day is broken up by three meals during which some residents require assistance, as well as optional morning and afternoon activities. Leisure activities offered at Sherbrooke range from physical activity, movie afternoons, and bingo sessions to music and entertainment (e.g., pizza nights). They generally take place at the care facility and are supported by several members of staff. Most residents require extensive assistance when attending to personal needs (e.g., getting dressed, bathing, or being taken to the toilet), and medical routines; this support mostly takes place in the early morning or replaces participation in optional leisure activities. Additionally, many residents also rely on

\footnotetext{
${ }^{1}$ http://www.edenalt.org/about-the-eden-alternative/
}

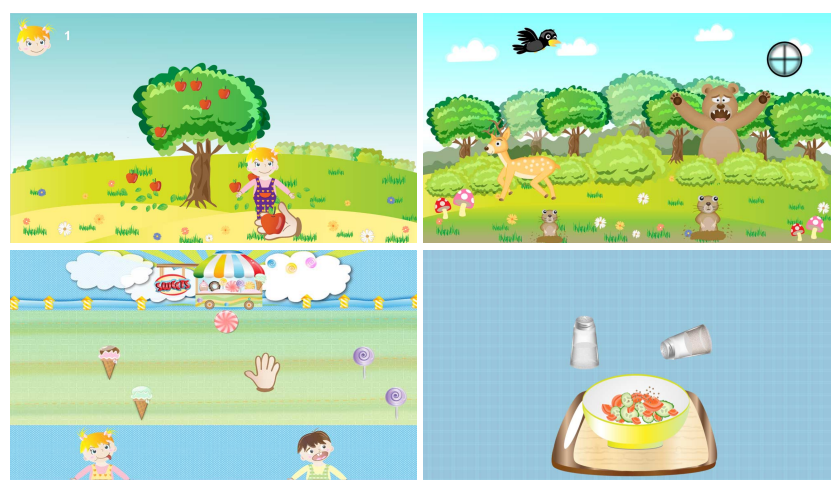

Figure 1-4. Screenshots of the four games applied in this study (top to bottom): Prairie Hunter, Candy Kids, Harvest Time, and Cooking Challenge.

assistance getting around the care facility, e.g., help to attend meals and social events.

\section{Games}

Keeping in mind the different groups of older adults at the two care facilities, we developed custom-designed games to ensure that participants would be able to gain access to video game play regardless of age-related changes and prior knowledge on how to play games. Additionally, we included commercially available video games to offer examples of more complex video game play.

\section{Custom-Designed Games}

We created four games based on design recommendations for games for older adults [10, 29]. All games can be played in single-player or competitive multiplayer mode. In Candy Kids, players have to feed a child different types of candy: items move across the upper area of the screen and can be fed to the child by moving the player avatar (represented by a virtual hand hand) over the food at the same time. Prairie Hunter invites players to set out on a virtual animal hunt. In order to increase their score, they have to hunt as many animals as possible. This is achieved by moving the crosshairs over the animal. Both Candy Kids and Prairie Hunter use pointing input, where the player's hand controls an avatar within the game. In Cooking Challenge, players have to prepare a salad. Ingredients are presented in the central area of the screen and have to be cut into pieces by imitating chopping movements and then pushed into the bowl by extending one's hand to the front. Once all ingredients are added, they have to be mixed which can be achieved by the imitation of a mixing gesture. Harvest Time invites players to cut down an apple tree using the same chopping gesture as in the previous game, and then shake the tree by moving one's hand left and right until an apple drops, and handing the apple to a girl who appears on screen by extending one's arm to the front. Cooking Challenge and Harvest Time implement gesture-based input that tries to mimic the real-world actions associated with the content of the games.

All games were implemented in C\# using Microsoft XNA Game Studio 4.0, and are played using the Microsoft 
Kinect. Our implementation invites players to use their strong hand to perform gestures and pointing actions to control the avatar.

\section{Commercially Available Games}

We included two commercially available games in our study. Both games are played on the Microsoft Xbox 360 and use the Kinect sensor as input device. Games were selected to represent casual Kinect gaming, and because input paradigms were likely to be accessible to older adults.

Kinect Sports. Kinect Sports offers a range of mini games that are based off traditional sports. In our study, we included Kinect Sports Bowling, a bowling simulation that is controlled by imitating real-world motions associated with bowling, e.g., swinging one's arm back and to the front to throw the ball. The game supports up to four players and allows for quick turn-taking similar to the actual sport. Additionally, games that were offered to residents included track and field (controlled through footbased input), and boxing (imitation of boxing movements with one's arms), in which two players directly compete against each other.

Kinect Adventures. Kinect Adventures is a full-body motion-based video game that consists of a number of minigames in which players are invited to apply their whole body to overcome in-game challenges in outdoor and fantasy settings. In our study, we included the following three games: In River Rush, the player stands on a raft and has to navigate down a river by jumping, ducking, and stepping to the sides. Space Pop is a game in which players need to flap their arms and move back and forth to float in a box that simulates a gravity-free environment to collect bubbles. In Rally Ball, players use a ball to hit targets displayed at the end of a box-sized playing area; after each strike, the ball bounces back to the player and he or she can use their body (i.e., arms and legs) to attempt another strike.

Games can be played in single player mode or with a partner; gameplay usually lasts for a couple of minutes. Both games use the Microsoft Kinect sensor as input device, if games are controlled with hand gestures, users are invited to use their strong hand.

\section{Procedure}

At each of the facilities, we offered a weekly supervised gaming session over the course of three months. In total, residents had the opportunity of participating in twelve supervised sessions. In addition, we invited residents to engage with the games outside the gaming sessions to understand whether games could serve as self-directed leisure activity. At the senior residence, supervised gaming sessions were paused for a month after the first two months to understand whether older adults would still engage in weekly play regardless of the availability of a facilitator. During this time, a member of staff was available to set up the gaming system, but did not facilitate play. We had planned to follow the same scheme at the care home, but were unable to do so because of the level of support required by participants. Players at the care home were unable to gain access to the set of commercially available games due to the complexity and nature of input (e.g., sequential actions necessary to play both Kinect and Wii Bowling, or participants with complex needs who would not be able to hold the Wii Remote - the home in fact owns a Wii, but residents do not use it due to accessibility barriers), and only interacted with the custom-designed games. Participants at the senior residence played the custom-designed games during the first month of the activity, and gradually transitioned to playing commercially available Kinect games. At the senior residence, gaming sessions were hosted in a community room. Games were displayed on a 40" TV screen. At the care home, gaming sessions were hosted in a media room on the floor that most residents lived on. Games were displayed on a 46" TV screen. Player input was tracked using the Kinect sensor.

This study was approved by the University of Saskatchewan behavioural ethics board, and was granted operational approval by Saskatoon Health Region. Additionally, it was approved by the internal review board at Sherbrooke Community Centre. Regarding consent procedures, we obtained informed written consent from participants at Brightwater Senior Living, and we obtained oral assent from participants at Sherbrooke Community Centre, which was witnessed and signed by a guardian acting on the behalf of the participant.

\section{Method}

We made observations and kept notes during each supervised gaming session, and at the end of each month, older adults participated in a focus group interview to reflect upon their gaming experience. Questions were designed to investigate the perceptions of video games among older adults (e.g., "What are your thoughts on the games that you played during the past month? Was there anything that you did not like?"). Additionally, we collected demographic information to gain insights into the personal background of participants, and examined their cognitive fitness using Folstein's Mini Mental State Exam (MMSE) [9] prior to their first gaming session. It was decided not to video or audio record gaming sessions to minimise the intrusiveness of our research upon this sensitive environment as much as possible.

\section{Participants}

Sixteen older adults (care home: six participants, one female, senior residence: ten participants, seven female) participated over the course of the study. Participants were recruited with the help of staff (social director and volunteer coordinator). At the care home, the average participant age was $73.5 \quad(\mathrm{SD}=4.18)$. All participants required walking assistance and were using wheelchairs. Mean scores for the Mini-Mental State Exam (MMSE) were at 13.3 points $(\mathrm{SD}=1.86)$, suggesting that all participants experience significant cognitive impairments. 
Only two participants had prior video game experience, mainly focusing on casual games. At the senior residence, average participant age was $79.2 \quad(\mathrm{SD}=4.80)$. Four participants used walkers, however, all of them were able to play the games without assistance. One participant used a wheelchair. Mean MMSE scores of $27(\mathrm{SD}=2.33)$ showed that none of the participants experienced significant cognitive impairments. Two participants had prior experience playing casual games on the computer or iPad.

\section{Data Analysis}

We report both quantitative and qualitative results and then provide explanations of our findings. Qualitative data were analyzed using inductive Thematic Analysis [6]. Interview results and observations were transcribed by the main investigator, analysis was carried out in line with the six phases of Thematic Analysis as proposed by Braun and Clarke [6]. We identified three main themes and nine subthemes. Table 1 shows an overview of the coding structure and relative frequencies at each facility.

\section{Results}

We organize our results around themes that were identified during analysis of qualitative data, and we contrast observations that we made at the two care facilities.

\section{Exploring The Game Experience of Older Adults}

The main theme that emerged during our analysis was the game experience of older adults, and we identified three subthemes: player experience and engagement is focused on the participants' perceptions of the games, competition and challenge explores how older adults perceive these core elements of games, and ownership of the gaming sessions summarizes participants' input that shaped the activity.

Player experience and engagement. Data on session attendance (Figure 5) show that seven of the ten participants at the senior residence and three out of six participants at the care home participated in at least half of the gaming sessions. The drop in attendance at the care home was caused by lack of support staff to help older adults attend gaming sessions, see theme "Support Before and After Play". Analysis of focus group interviews shows that older adults who continued to participate in our study generally consider motion-based games an enjoyable activity, pointing out that "it's fun to do something physical" (P2, senior residence); many participants at the senior residence explained that they enjoyed playing video games that simulated real-world activities they can no longer participate in. When asked about differences between custom-designed mini games and commercially available games, players at the senior residence pointed out that the mini games were appropriate to get more familiar with video games, but that the long-term appeal of more complex commercially available applications was bigger, which is also reflected by their choice of games throughout the gaming sessions where participants increasingly requested to play Kinect Sports Bowling. Participants at the
Table 1. Structure and relative code distribution within each theme at the care home $(\mathrm{CH})$ and senior residence (SR).

\begin{tabular}{llcc} 
Theme & Subtheme & $\mathbf{\% C H}$ & $\mathbf{\% S R}$ \\
\hline $\begin{array}{l}\text { Game } \\
\text { Experi- } \\
\text { ence }\end{array}$ & Enjoyment \& Engagement & 39 & 33 \\
\cline { 2 - 4 } & Challenge \& Competition & 44 & 49 \\
\cline { 2 - 4 } $\begin{array}{l}\text { Social } \\
\text { Setting }\end{array}$ & Ownership & 17 & 18 \\
\cline { 2 - 4 } & Player Inhibition & 55 & 18 \\
\cline { 2 - 4 } & Disruptive Interactions & 45 & 23 \\
\hline \multirow{3}{*}{ Support } & Community Value & 0 & 59 \\
\cline { 2 - 4 } & Group Support & 0 & 47 \\
\cline { 2 - 4 } & Facilitator Support & 50 & 47 \\
\hline
\end{tabular}

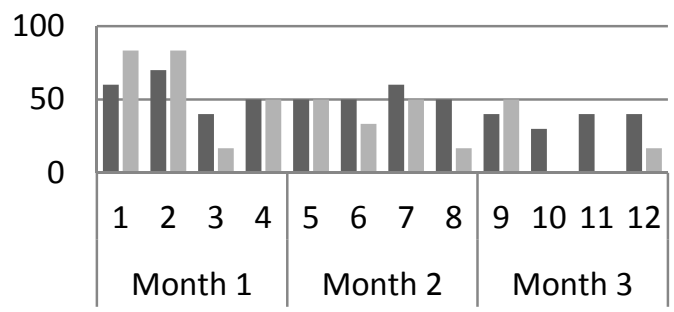

Senior Residence $\quad$ Nursing Home

Figure 5. Attendance rates of supervised gaming sessions (in percent per week) at the senior residence and nursing home.

care home preferred playing the hunting game due to the appeal of the theme and simplicity of game input; participants at both facilities reported physical fatigue as a result of motion-based play.

To better understand the game experience of older adults, we followed up with participants who decided to quit the activity. Many participants who permanently dropped out of the gaming sessions pointed out that they simply lost interest in playing video games. One participant stated she found out "it simply wasn't my cup of tea" (P5, care home), another one outlined she felt she could "do better things with my time" (P7, senior residence), outlining the need for further research to explore suitable game concepts for older adults. Some players commented on being bored during the course of the session, particularly if games did not support quick turn-taking (e.g., Kinect Adventures), or if changing between players took too much time (particularly at the care home due to prevalence of age-related impairments), with one participant falling asleep during the gaming session while waiting his turn. Finally, health issues were a barrier to continued participation for participants at both facilities. While some participants only experienced minor health problems (e.g., feeling tired or seasonal illness) that caused them to miss single sessions and allowed them to join up with the group after their recovery, three participants who showed great interest in the initial gaming sessions (one at the care home, two at the senior residence) permanently dropped out due to major health problems (e.g., long-term hospitalization). 
Game challenge and competition. Observations made during our study revealed that challenge and competition play an important role in the gaming experience of the participants at both facilities, suggesting that overcoming challenge - a core aspect of video games - can be a strong source of enjoyment, but also induce frustration and discouragement. Many participants pointed out that they enjoy a feeling of accomplishment when successfully overcoming in-game challenges. At the senior residence, accomplishment mostly stemmed from competition with other players, but also from some individual achievement (e.g., throwing a strike in bowling). At the care home, accomplishment was associated with successfully being able to engage with the games; challenge was sometimes introduced by players attempting to beat themselves by trying to achieve higher scores than in previous sessions. At both facilities, participants were interested in their scores as a measure of success, and cheered about accomplishments. Particularly at the senior residence, players encouraged each other to do better, commented on individual accomplishments, and clapped and cheered if a player did well. We observed an incident where a stronger player at the senior residence dropped her score in bowling when another participant couldn't get the hang of the game. This reduced the negative experience for other player and allowed her to ease back into play.

Our analysis also revealed negative aspects of the competitive nature of play. Participants at both facilities showed frustration and disappointment when not performing as well as expected. This is particularly difficult in the context of social play. For example, one player at the care home brought along a friend to a gaming session, and got visibly angry when he could not keep up with her. Likewise, participants at the senior residence became frustrated about a particularly strong player when engaging with the custom-designed mini games and refused to compete against her in future sessions. We also observed potentially harmful forms of discouragement as a result of competition. Comments of weaker players at the senior residence revealed signs of self-consciousness after watching stronger players, and some were discouraged by large score gaps. Two female participants in particular were very hard on themselves, reflecting upon their own skills and the impact of age-related changes, with one of them commenting that "I can hardly walk, how can I bowl?"

Ownership of gaming experience. At both facilities, participants took ownership of their gaming experience. While players at the care home mostly expressed preferences in some mini games over others, players at the senior residence did not only choose preferred games from the pool offered in the context of the activity, but also inquired about other commercially available games that might be suitable for them. In particular, two participants had a personal interest in winter sports and considered the purchase of Kinect Winter Stars after it had been identified as a suitable candidate. Furthermore, players at the senior residence gradually extended the duration of the gaming sessions beyond the facilitated time, and considered the introduction of a second session later in the week.

\section{Implications of Playing Games in a Social Setting}

Our analysis revealed that playing games together with a group of others in the context of a programmed activity had a profound impact on the experience of the participants. In our data, we identified three recurring subthemes: The inhibition of player engagement as a result of the group setting, and disruptive interactions between players that negatively influenced the experience of individuals, but also the large potential that games have to build community between players.

Inhibition of player engagement. Participants at both facilities felt that playing video games in front of their peers was intimidating. Players at the senior residence were mostly concerned about the process of learning how to play and needed encouragement during the first gaming sessions. During the focus group interview, one participant noted that she would have been more comfortable during this stage without the presence of others, stating she felt as if "everybody was watching, you know they are there" (P3, senior residence). Another participant had a discouraging experience when first playing Kinect Adventures. One of the mini games required him to lift both arms; however, he was unable to reach high enough, exposing his age-related impairment in front of other group members. Likewise, the group setting caused major problems for participants at the care home. Observations revealed that players found it extremely difficult to learn how to interact with the games in front of a group and were self-conscious about their ability to play games, which is backed up by interview results where one participant was pointing out that you "gotta work your way into the games, then it's okay [to play with others]". Two participants specifically suggested that they would prefer individual gaming sessions, and one participant who continued to participate in these individual sessions outlined that he enjoyed playing on his own, because he could play at his own pace and "not worry about what others are doing" ( $\mathrm{P} 2$, care home).

Disruptive interactions. The group setting occasionally caused friction among participants at both facilities. We observed disruptive interactions between individual participants, within the group, and as a result of the general setting. Participants at the care home mostly experienced basic communication problems due to the impact of agerelated changes. For example, at one point, a participant with a hearing impairment tried to communicate with another participant who had a speech impairment, both growing increasingly frustrated about the inability of the other person to understand what they were trying to express. Disruptive interactions between individuals at the senior residence mostly took place on a higher level, e.g., one participant attending one of the gaming sessions with bare feet, offending another participant who felt the attire 
was inadequate for the group event. A quick conversation revealed that the barefoot participant was unwilling to put on shoes, which caused the other person to leave the session in disagreement. Finally, participants at the senior residence became openly frustrated about other players who did not understand the turn-taking process and therefore acted out of turn (e.g., when playing Kinect Sports Bowling) on multiple occasions. During one session, this led to the early departure of one participant. However, emerging conflict was usually resolved through group intervention.

Community value. In contrast to the often-difficult experiences of social play at the care home, gaming sessions at the senior residence revealed great potential to build community over the course of the programmed activity. As the residence was a newly established facility, participants of the gaming sessions did not know each other prior to participation in the activity, and we observed that shared play served as a catalyst for community building. We observed how participants gradually became more familiar with each other over the weeks (e.g., learning each other's names), and communication throughout the sessions evolved beyond play and moved on to topics not directly related to the games. Within the first month, a core group of four participants emerged that would continuously engage in the sessions. We observed that this group grew closer every week (e.g., sharing personal stories, and making arrangements to have lunch together at the end of the session), but at the same time remained welcoming and supportive of new members, actively encouraging the participation of other residents in the gaming sessions. Additionally, other residents, staff, and guests frequently dropped into gaming sessions to spectate, suggesting that the activity may also provide value for persons not actively participating in play. Feedback collected during focus groups at the senior residence supports the appeal of gaming sessions as social activity. Participants highlighted that they enjoyed the opportunity to meet other residents, pointing out that "it's being involved that feels good" (P8, senior residence) and that "it's good fun to be together and participate" ( $\mathrm{P} 3$, senior residence).

\section{Forms of Support Before and During Play}

Another theme that emerged during analysis is focused around support that older adults require to participate in gaming sessions. We identified three subthemes - staff support and facilitator support, which are both external sources initially required at both care facilities, and group support, which focuses on participant efforts of taking ownership of the gaming sessions at the senior residence.

Staff support. At each of the care facilities, different levels of staff support were necessary to support the gaming sessions. At the senior residence, staff activity was limited to the recruitment of participants by announcing the activity to residents, and the support of an additional month of play by setting up the gaming system, but then withdrawing from the sessions and leaving them to the participants. In contrast, staff was required before all gaming sessions at the care home. Due to the prevalence of age-related impairments, participants depended on the support of staff to be able to attend the gaming sessions: many older adults at the care home needed help when navigating their environment (e.g., to attend meals). If staff was unavailable due to other tasks (e.g., supervising community outings), residents could not be helped to transfer into their wheelchairs, and moved to the media room and could therefore not participate in gaming sessions, which is reflected by low attendance rates of gaming sessions during month three, where the social coordinator who had previously supported residents was on leave, and no other support staff was available (Figure 5).

Facilitator support. Facilitator support was offered for all of the gaming sessions. At both facilities, participants received an introduction to the games, and the facilitator was available to set up the gaming system and guide participants through gaming sessions similar to support available during other activities (e.g., a fitness instructor during physical activity sessions). While participants at the senior residence mostly needed support when switching between games (e.g., help navigating complicated menu structures of Kinect games) and some support organizing the session (e.g., assistance to organize turn-taking through verbal cues when feedback of the game is unclear, especially when playing Kinect Sports Bowling), participants at the care home heavily relied on the facilitator over the entire session, needing assistance when swapping players (e.g., pushing wheelchairs), but also continuous help while engaging with the games (e.g., reminders on how to interact, the goal of the game, etc.).

Group support. At the senior residence, facilitator support throughout the gaming sessions was gradually replaced by group support. This is another example of how participants took ownership of gaming sessions, actively explaining how to play the games to persons that decided to participate in the activity after the initial introduction. Throughout the sessions, the group also played a role in supporting weaker players, e.g., by reminding them of interaction paradigms: one participant with early onset dementia repeatedly forgot part of the interaction sequence necessary to play Kinect Sports Bowling, and the group patiently reminded her of the necessary steps. Additionally, stronger players also shared strategies for improvement with the group; particularly when playing Kinect Sports Bowling and Boxing, ways for improvement were frequently debated. Finally, the group also took over staff support that would have been necessary to remind participants of the activity prior to session start by making their rounds through the residence and informing others about the activity.

\section{CORE CHALLENGES IN THE DEPLOYMENT OF GAMES IN LONG-TERM CARE}

This section provides insights into the core challenges related to the development and deployment of video games 
in residential care that stem from the three main themes identified in our work. We particularly focus on differences that we observed between participants at the care home and senior residence that emerged as a result of different levels of age-related changes in cognitive and physical abilities.

\section{(1) Considering the Ambiguity of Social Play}

Our results suggest that playing games in a social setting introduces challenges and opportunities for older adults and their peers. While the opportunity to socialize with other residents represented one of the biggest pulls to participate at the senior residence, having to play games in a group created additional barriers for players at the nursing home. It is therefore important that game designers understand the ambiguity of social play. On the one hand, it is an opportunity for older adults to come together as a group, allowing older adults to enjoy the company of others while watching them play. On the other hand, social play and the presence of others can be a barrier for persons who experience higher levels of age-related changes and impairments, have little confidence in their ability of playing games, or are not comfortable being observed when learning how to play. While a lot of research highlights the potential benefits of social play, particularly in the context of games for older adults [21], it is important that designers understand the risk of games exposing the vulnerability of institutionalized older adults by revealing individual agerelated changes, which is particularly challenging when working with persons with cognitive or physical impairments. In this context, game developers need to rethink traditional design principles: challenging players and pushing limits in multiplayer settings may be appealing for active older adults, but can turn into a major barrier for players who are challenged to adapt to cognitive and physical changes later in life.

\section{(2) Providing Adequate Levels of Challenge}

Previous work has identified game challenge as one of the core motivations for active older adults to engage with games [8]. Our work suggests that challenge can indeed function as a source of long-term player motivation. Regardless of cognitive and physical abilities, participants at both facilities enjoyed and sought out the feeling of accomplishment experienced after successfully overcoming challenging in-game tasks. However, differences in player abilities and their impact on play also underline the importance of offering adequate levels of challenge. Game challenge also functioned as a source of frustration and discouragement when players were unable to meet the goals specified in the game, or unable to match the scores of their co-players. This highlights a risk for the emotional wellbeing of players if failure is perceived as a consequence of age-related changes rather than weak game balancing. However, frustration at failure is a natural consequence of participating in challenging activities, so game designers cannot design to avoid these types of experiences entirely. Therefore, designers must be creative in designing challenges that do not repeatedly present frustrating and discouraging experiences while accommodating a wide range of cognitive and physical abilities among players.

(3) Embedding Games in the Context of Long-Term Care Our study shows that the long-term deployment of games in residential care facilities requires considerations that take into account aspects of daily life in residential care facilities, particularly the availability of staff or volunteers to support residents in attending and setting up gaming sessions, and the ability of residents of taking ownership of gaming sessions. Previous studies [10, 17] have discussed the role of staff members in the basic upkeep and setup of games. Our study shows that depending on the nature of care facility, more specialized help is often necessary. While participants at the senior residence took the lead in facilitating gaming sessions beyond system setup, participants at the care home who experienced age-related cognitive and physical impairments required additional assistance to be able to participate in play. Specifically, if older adults require the support of staff to be able to attend gaming sessions as observed at the care home, care facilities must provide higher levels of assistance in order for gaming sessions to function as accessible and successful activities for their residents. However, games could be designed to better accommodate group support (i.e., independent older adults leading sessions) by offering accessible setup routines, increasing group ownership and reducing the need of staff support.

\section{DISCUSSION}

This paper presents a qualitative enquiry into the long-term deployment of motion-based video games for older adults in residential care. Our results suggest that playing video games in the context of a weekly activity is enjoyable and empowering for independent older adults, but difficult when persons experience complex age-related changes and impairments. These changes impact a person's ability to engage with games for reasons beyond simple game accessibility, for example, influencing how older adults view the context of play and how much assistance they require. In this section, we discuss the implications of our findings. We summarize key considerations in designing video games to be deployed in residential care, and we discuss the role of games in the lives of older adults.

\section{Designing for Older Adults in Residential Care}

Previous studies suggest that accessible games for institutionalized older adults can be created by accounting for age-related changes $[10,11]$; while these considerations remain valid in the context of our work, results suggest that this is only a first step toward the successful deployment of games in long-term care, and that a more comprehensive approach to development is necessary. Age-related changes and impairments do not only influence whether basic gameplay is accessible, but how older adults see themselves in the gaming context, how they perceive the participation of others, and how they respond to in-game challenge. These aspects need to be reflected by game concepts; the range of skills and abilities among older adults in 
residential care is wide; to be successful, games need to engage players of all abilities, meaning that games may have to be tailored towards specific groups.

\section{Understanding the Role of Games in Residential Care}

Our study provides new insights into the role of games for older adults in residential care. In the following sections, we discuss the value of games as a self-directed leisure activity, and we explore whether games could serve as a means of re-introducing challenge in the lives of older adults in long-term care.

\section{Games as a Self-Directed Leisure Activity}

Previous research has suggested that video game playing can function as a valuable self-directed leisure activity for older adults in residential care. The findings of our study suggest a more nuanced understanding is needed of the audience targeted by a game in order to ensure that those benefits are experienced. Specifically, for people with more advanced age-related changes and impairments, the deployment of games becomes increasingly challenging and requires extensive support of nursing staff both prior to play and during gaming sessions. For these participants, games cannot be considered an independent activity to participate in without the support of others. This must be considered carefully when evaluating the benefits of games compared to other supervised leisure activities in care facilities.

\section{Games as a Source of Challenge in Late Life}

For many people, overcoming challenges and accomplishing goals are important contributors to their self-efficacy and self-esteem [22]. In late life, we can no longer accomplish many of the tasks from which we once drew a sense of achievement. Research suggests that older adults who adapt and find new sources of challenge and self-esteem have better mental health [5]. However, residential care environments rarely present challenging activities, to older adults. Our work shows that games may be a means of reintroducing challenge in the lives of older adults in long-term care: if games provide appropriate levels of challenge, they are a means of enabling older adults to experience accomplishment, can foster competition, and allow older adults to experience competence within their peer group.

\section{Satisfying Needs through Games}

Ryan et al. [25] have demonstrated that video games can be a means of needs satisfaction because they allow players to experience competence, autonomy, and relatedness in combination with an experience of immersion and intuitive control. Examining our work through the lens of needs satisfaction sheds an interesting light on our findings. In residential care, games offer an opportunity to experience competence; however, in situations where games highlight age-related changes, they may actually reduce perceived competence. Games offer players the opportunity to socialize and satisfy their need for relatedness; however, the embarrassment of not keeping up with peers might prevent the satisfaction of this need. Finally, giving players in residential care the opportunity to choose participate in play can help satisfy their need for autonomy; however, presenting games as a leisure option where people feel like they are not participating under their own volition can reduce this experience. Therefore, to allow older adults in long-term care to leverage games to satisfy their core needs for overall well-being, the unique effects of age-related changes and challenges associated with the context of long-term care must be considered.

\section{LIMITATIONS AND FUTURE WORK}

There are limitations to the work discussed in this paper; our study is of an exploratory nature and only includes a small number of participants at both care facilities; our findings presented in this paper should be viewed in this light. In this context, our study provides several opportunities for future work. First, studying the long-term gaming behaviour of older adults in a quantitative context would be valuable to expand on the findings of our qualitative enquiry. Addressing the question of how games could be designed to facilitate the involvement of staff, and how care facilities could best be supported in the deployment of games is an important step towards supporting care facilities wishing to turn video games into a valuable leisure activity for their residents. Additionally, conducting research with a detailed focus on social aspects of play among older adults - for instance, the role of competition among players - could provide further insights into how games can successfully be applied to strengthen social relationships among care home residents.

\section{CONCLUSION}

Understanding how older adults in residential care experience video game play in the context of a programmed activity is important to arrive at a better understanding of how games can be applied to benefit this audience. Our results suggest that playing games in a social setting can be an empowering and appealing experience for independent older adults, but may expose the vulnerability of those who experience age-related impairments. Generally, our work shows that games offer an opportunity of diversifying leisure activities in long-term care, and may be leveraged to promote positive self-views among older adults. However, to offer these benefits, the research community needs to be aware of the risks of play for vulnerable older adults, and carefully design games to meet their needs. This is particularly important when evaluating the value of games for improving the quality of life of older adults, and when creating games with a purpose beyond entertainment, such as therapy and rehabilitation.

\section{ACKNOWLEDGMENTS}

We would like to thank Sherbrooke Community Centre and Brightwater Senior Living and all study participants for supporting our research.

\section{REFERENCES}

1. Alankus, G., Lazar, A., May, M., and Kelleher, C. Towards Customizable Games for Stroke Rehabilitation. In: Proc. of CHI 2010, ACM Press (2010), 2113-2122. 
2. Anderson-Hanley, C., Arciero, P.J., Brickman, A.M., Nimon, J.P., Okuma, N., Westen, S.C., Merz, M.E., Pence, B.D., Woods, J.A., Kramer, A.F., and Zimmerman, E.A. Exergaming and Older Adult Cognition: A Cluster Randomized Clinical Trial. Am J Prev Med 42, 2 (2012), 109-119.

3. Bateni, H. Changes in balance in older adults based on use of physical therapy vs the Wii Fit gaming system: a preliminary study. Physiotherapy (2011).

4. Blythe, M., Wright, P., Bowers, J., Boucher, A., Jarvis, N., Reynolds, P., and Gaver, B. Age and Experience: Ludic engagement in a residential care setting. In: Proc. of DIS 2010, ACM Press (2010), 161-170.

5. Brandstädter, J., and Greve, W. The Aging Self: Stabilizing and Protective Processes. Developmental Review, 42 (1994), 52-80.

6. Braun, V., and Clarke, V. Using Thematic Analysis in Psychology. Qualitative Research in Psychology 3, 2 (2006), 77-101.

7. Chen, Y., Ngo, V., and Park, S.Y. Caring for Caregivers: Designing for Integrality. In: Proc. of CSCW 2012, ACM (2012), 91-102.

8. De Schutter, B. Never Too Old to Play: The Appeal of Digital Games to an Older Audience. Games and Culture 6, 2 (2011), 155-170.

9. Folstein, M.F., Folstein, S.E., and McHugh, P.R. "MiniMental State" - A Practical Method for Grading the Cognitive State of Patiens for the Clinician. J Psychiat Res 12, (1975), 189-198.

10. Gerling, K.M., Livingston, I.J., Nacke, L.E., and Mandryk, R.L. Full-Body Motion-Based Game Interaction for Older Adults. In: Proc. of CHI 2012, ACM Press (2012).

11. Gerling, K.M., Mandryk, R.L., and Kalyn, M.R. Wheelchair-Based Game Design for Older Adults. In: Proc. of ASSETS 2013, ACM Press (2013).

12. Harley, D., Fitzpatrick, G., Axelrod, L., White, G., and McAllister, G. Making the Wii at home: game play by older people in sheltered housing. HCI in Work and Learning, Life and Leisure, Springer (2010), 156-176.

13. Jung, Y., Li, J.K., Janissa, N.S., Gladys, W.L.C., and Lee, K.M. Games for a better life: effects of playing Wii games on the well-being of seniors in a long-term care facility. In: Proc. of IE '09, ACM Press (2009).

14. Khan, D.U., Siek, K.A., Meyers, J., Haverhals, L.M., Cali, S., and Ross, S.E. Designing a Personal Health Application for Older Adults to Manage Medication. In: Proc. of IHI '10, ACM (2010).

15. Kientz, J.A., Patel, S.N., Jones, B., Price, E.D., Mynatt, E.D., \& Abowd, G.D. The Georgia Tech Aware Home. In: EA of CHI 2008, ACM Press (2008), 3675-3680.
16. Lindley, S.E., Harper, R., and Sellen, A. Designing for Elders: Exploring the Complexity of Relationships in Later Life. In: Proc. of HCI 2008, ACM (2008), 77-86.

17. Marston, H.R., Greenlay, S., and van Hoof, J. Understanding the Nintendo Wii and Microsoft Kinect consoles in long-term care facilities. Technology and Disability 25, 2 (2013), 77-85.

18. Mueller, F., Edge, D., Vetere, F., Gibbs, M.R., Agamanolis, S., Bongers, B., and Sheridan, J.G. Designing Sports: A Framework for Exertion Games. In: Proc. of CHI 2011, ACM (2011), 2651-2660.

19. Müller, C., Neufeldt, C., Randall, D., and Wulf, V. ICTDevelopment in Residential Care Settings: Sensitizing Design to the Life Circumstances of the Residents of a Care Home. In: Proc. of CHI 2012, ACM Press (2012).

20. Nap, H.H., de Kort, Y.A.W., and IJsselteijn, W.A. Senior gamers: Preferences, motivations and needs. Gerontechnology 8, 4 (2009), 247-262.

21. Pedell, S., Beh, J., Mozuna, K., and Duong, S. Engaging Older Adults in Activity Group Settings Playing Games on Touch Tablets. In: Proc. of OzCHI'13, ACM (2013), 477-480.

22. Pelham, B.W., and Swann, W.B. From Self-Conceptions to Self-Worth: On the Sources and Structure of Global Self-Esteem. Journal of Personality and Social Psychology 57, 4 (1989), 672-680.

23. Penedo, F.J., and Dahn, J.R. Exercise and well-being: a review of mental and physical health benefits associated with physical activity. Curr Opin Psychiatry, 18 (2005).

24. Rice, M., Tan, W.P., Ong, J., Yau, L.J., Wan, M., and Ng, J. The Dynamics of Younger and Older Adult's Paired Behaviour When Playing an Interactive Silhouette Game. In: Proc. of CHI 2013, ACM (2013), 1081-1090.

25. Ryan, R., Rigby, C., and Przybylski, A. Motivational pull of video games: A self-determination theory approach. Motivation and Emotion, 30 (2006), 347-365.

26. Theng, Y.-L., Chua, P.-H., and Pham, T.P. Wii as Entertainment and Socialisation Aid for the Mental and Social Health of the Elderly. In: EA of CHI 2012, ACM (2012), 691-702.

27. Ulbrecht, G., Wagner, D., and Gräßel, E. Exergames and Their Acceptance Among Nursing Home Residents. Activities, Adaptation \& Aging 36, 2 (2012), 93-106.

28. Uzor, S., and Baille, L. Investigating the Long-Term Use of Exergames in the Home With Elderly Fallers. In: Proc. of CHI 2014, ACM (2014), 2813-2822

29. Vanden Abeele, V. and De Schutter, B. Meaningful Play in Elderly Life. In: Proc. of ICA 2008, (2008). 